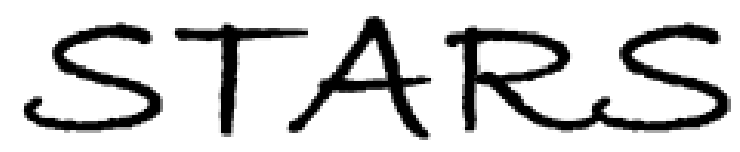

University of Central Florida

STARS

$1-1-2013$

\title{
Label-free cell phenotypic assessment of the molecular mechanism of action of epidermal growth factor receptor inhibitors
}

Huayun Deng

Chaoming Wang

University of Central Florida

Ye Fang

Find similar works at: https://stars.library.ucf.edu/facultybib2010

University of Central Florida Libraries http://library.ucf.edu

This Article is brought to you for free and open access by the Faculty Bibliography at STARS. It has been accepted for inclusion in Faculty Bibliography 2010 s by an authorized administrator of STARS. For more information, please contactSTARS@ucf.edu.

\section{Recommended Citation}

Deng, Huayun; Wang, Chaoming; and Fang, Ye, "Label-free cell phenotypic assessment of the molecular mechanism of action of epidermal growth factor receptor inhibitors" (2013). Faculty Bibliography 2010s. 3886.

https://stars.library.ucf.edu/facultybib2010/3886

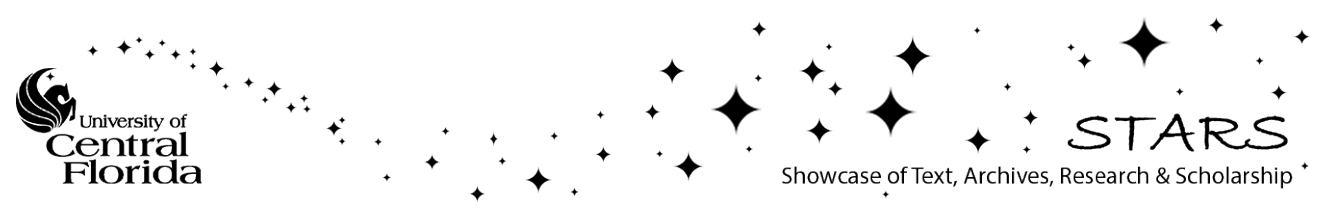


Cite this: RSC Advances, 2013, 3, 10370

Received 25th January 2013, Accepted 12th April 2013

DOI: 10.1039/c3ra40426a

www.rsc.org/advances

\title{
Label-free cell phenotypic assessment of the molecular mechanism of action of epidermal growth factor receptor inhibitors
}

\author{
Huayun Deng, ${ }^{a}$ Chaoming Wang ${ }^{\mathrm{ab}}$ and Ye Fang*a
}

\begin{abstract}
Epidermal growth factor receptor (EGFR) is the target of several clinically approved tyrosine kinase inhibitor (TKI) drugs including gefitinib and erlotinib in the treatment of cancer. Multiple mechanisms have been implicated in the clinical features of these drugs. However, little is known about the molecular mechanism of action of these drugs at the whole cell level. Here we applied a label-free biosensor-enabled dynamic mass redistribution (DMR) assay to assess the molecular mechanism of action of three EGFR inhibitors, gefitinib, erlotinib and AG1478, to alter the EGFR signaling in A431 and HT-29, two native cancer cell lines expressing the EGFR. The whole-cell DMR assays with the persistent inhibitor treatment showed that all inhibitors dose-dependently inhibited the EGFR signaling in both cell lines, but generally displayed higher potency in A431 than HT-29 cells. The DMR assays with the inhibitor washout showed that the washout unexpectedly increased the potency of gefitinib and AG-1478 to inhibit the EGFR signaling in A431, but slightly decreased the potency of all three inhibitors in HT29. The DMR assays under microfluidics showed that the removal of the inhibitors using buffer perfusion resulted in a timedependent recovery of EGF signaling that is slower in A431 than HT-29 cells. In contrast, DMR assays under microfluidics showed that the removal of reversible competitive antagonists led to the full recovery of the signalling of two distinct G protein-coupled receptors (GPCRs), the $\beta_{2}$-adrenergic receptor in A431 and the GPR35 in HT-29 cells. Together, our results suggest that for EGFR inhibitors their uptake and retention, rather than binding kinetics, dominate their label-free cell phenotypic efficacy; however, for GPCR antagonists the binding characteristics are critical to the inhibitory effects. This study also implicates the potential of DMR assays under different simulation conditions for elucidating the cell phenotypic pharmacology, in particular transporter-related drug resistance, of kinase inhibitor drugs.
\end{abstract}

\section{Introduction}

The epidermal growth factor receptor (EGFR) family of receptor tyrosine kinases consists of four members including EGFR, erbB2 (HER2), erbB3 and erbB4. With the exception of HER2, all bind to receptor-specific ligands. Agonist binding facilitates either homo- or hetero-dimerization between different receptors of the EGFR family, leading to the activation of a multitude of signaling cascades via their tyrosine kinase activity. ${ }^{1-3}$ EGFR plays a critical role in the control of cell proliferation, differentiation, and survival. Abnormalities in signaling of the EGFR pathway have been found in a wide range of cancers, including lung, breast, and colon cancers. EGFR activation in many epithelial malignan-

${ }^{a}$ Biochemical Technologies, Science and Technology Division, Corning Incorporated, Corning, NY 14831, United States of America. E-mail: fangy2@corning.com; Fax: +1 607-974-5957

${ }^{b}$ NanoScience Technology Center, Department of Mechanical Materials and Aerospace Engineering, University of Central Florida, Orlando, Florida, United States of America cies has been shown to be a result of overexpression of ligands and receptors, EGFR gene gain, or activating mutations; and EGFR activation appears important in tumour growth and progression. ${ }^{4-6}$ The inhibitors of EGFR and/or HER2 signaling have been extensively evaluated in the clinic and shown to have therapeutic benefits for certain cancer patients. ${ }^{6-8}$ The marketed EGFR antagonist drugs to date include monoclonal antibodies, such as cetuximab and panitumumab, and small molecule tyrosine kinase inhibitor (TKI) drugs, such as erlotinib, gefitinib, lapatinib and icotinib, that target the catalytic domain of the EGFR. ${ }^{6-9}$

Enormous efforts have been attempted to elucidate the molecular mechanism(s) of action underlining the clinical features including efficacy, ${ }^{4-7}$ resistance $^{10-12}$ and safety ${ }^{13}$ profiles of EGFR inhibitor drugs. The clinical efficacy of these inhibitors has been attributed to their ability to inhibit the activation and signaling pathways of overexpressed EGFR or its activating mutants via reversible competitive binding with MgATP to the intracellular protein tyrosine kinase domain of the receptor. ${ }^{4-7}$ Factors that contribute to the resistance 
include expression of activating mutants of EGFR downstream signaling cascades such as $\mathrm{K}-\mathrm{RAS},{ }^{11}$ the expression of inhibitor-insensitive activating receptor mutants, ${ }^{12}$ overexpression of drug transporters such as breast cancer resistance protein (BCRP/ABCG2), ${ }^{14}$ or residues of cancer stem cells. ${ }^{15}$ Recently, the binding kinetics, in particular drug residence time (reciprocal of the off rate), have been implicated in the in vitro and in vivo efficacy and other profiles of drugs for many classes of targets. ${ }^{16-20}$ For EGFR TKIs, both on and off rates of the binding have been postulated to impact their efficacy to block EGFR signaling. ${ }^{21-23}$ However, little is known about the molecular mechanism of action of the EGFR inhibitors to block receptor signaling in real time and at the whole cell signaling network level.

Here we report an assessment of the molecular mechanism of action of three inhibitors to inhibit the EGFR signaling at the whole cell level using label-free biosensor-enabled dynamic mass redistribution (DMR) assays under different assay conditions. DMR assay is a whole cell phenotypic assay that offers a holistic and real-time view of the drug action through the receptor in native cells. ${ }^{24-27}$ Using DMR assays we showed that the uptake and retention of these TKI inhibitors are more important to their whole cell efficacy for inhibiting EGFR signaling than their binding kinetics.

\section{Materials and methods}

\section{Materials}

Epidermal growth factor (EGF) was obtained from Bachem (Torrance, CA, USA). AG1478, CID 2745687, gefitinib (Iressa), isoprotenerol, sotalol, and zaprinast were obtained from Tocris Chemical Co. (St. Louis, MO, USA). Erlotinib (Tarceva) was obtained from Santa Curz Biotechnology Inc. (Santa Curz, CA, USA). Polydimethylsiloxane (PDMS) was obtained from Dow Chemical (Midland, MI, USA). Epic $\mathbb{R}$ 384-well biosensor cell culture compatible microplates were obtained from Corning Incorporated (Corning, NY, USA). Human colorectal adenocarcinoma HT-29 and human epidermoid carcinoma A431 cell lines were obtained from American Type Cell Culture (Manassas, VA, USA).

\section{Cell culture}

The HT-29 cells were cultured in McCoy's 5a Medium Modified supplemented with $10 \%$ fetal bovine serum, $4.5 \mathrm{~g} \mathrm{~L}^{-1}$ glucose, $2 \mathrm{mM}$ glutamine, and antibiotics at $37{ }^{\circ} \mathrm{C}$ under air $/ 5 \% \mathrm{CO}_{2}$. The A431 cells were cultured using Dulbecco's Modified Eagle's Medium (DMEM) supplemented with 10\% fetal bovine serum, $4.5 \mathrm{~g} \mathrm{~L}^{-1}$ glucose, $2 \mathrm{mM}$ glutamine, and antibiotics in a humidified $37{ }^{\circ} \mathrm{C} / 5 \% \mathrm{CO}_{2}$ incubator.

For DMR assays in microplate, the cells were cultured in Epic ( ) biosensor cell culture compatible microplates for one day. The initial seeding density was 25000 and 32000 cells well $^{-1}$ for A431 and HT-29, respectively. After culture for $\sim 20$ $\mathrm{h}$ the cells reached a confluency of $\sim 95 \%$. A431 cells were further subject to overnight starvation under serum-free medium.
For DMR assays under microfluidics the cells were cultured in a PDMS-based microfluidic biosensor device. The microfluidic device was fabricated using a method described previously. ${ }^{28,29}$ Briefly, a PDMS pre-polymer solution containing a mixture of PDMS oligomers and a reticular agent with 10:1 mass ratio (Sylgard 184 Kit, Dow Corning Corp., Midland, MI, USA) was cast onto patterned silicon wafers, and cured at room temperature for about $24 \mathrm{~h}$ to minimize shrinkage after curing. Afterwards, the PDMS replicas obtained were punched to generate inlet and outlet holes, and then aligned and reversibly bonded onto the top of the biosensor inserts. The microfluidic biosensor device has a $3 \times$ 4 array of functional RWG biosensors, each having a dimension of $2 \times 2 \mathrm{~mm}$, and a $3 \times 4$ array of microfluidic chambers, each having three inlets and one outlet. The distance from an inlet to the outlet is $9 \mathrm{~mm}$, the central width of the chamber $5 \mathrm{~mm}$. To ensure appropriate cell culture and minimize the effect of any non-specific absorption of ligand molecules to the top PDMS surface of the microchamber on the cells, the height of the microchannel was set to be $200 \mu \mathrm{m}$. The total volume required to fill up a chamber is $6 \mu \mathrm{l}$. Before cell culture, the device, tubing (Tygon S-54-HL, Saint-Gobain Performance Plastics, Akron, OH, USA) and syringes $(500 \mathrm{ml}$, gas tight 1700 series, Hamilton, Reno, NV, USA) were sanitized with $70 \%$ ethanol and dried using nitrogen flow. Immediately, $4 \times 10^{4}$ cells suspended in $6 \mu \mathrm{l}$ of the culture medium were injected into each chamber. Cells were allowed to seed via 30 min incubation at room temperature. Tubing was then plugged into the microchamber inlets and was connected to syringes connecting to a syringe pump (Model: SP230IW; World Precision Instruments, Sarasota, FL, USA). The cells were then cultured at $37{ }^{\circ} \mathrm{C} / 5 \% \quad \mathrm{CO}_{2}$ with a continuous perfusion of the culture media at a flow rate of $5 \mu \mathrm{h} \mathrm{h}^{-1}$. After culture for $\sim 20 \mathrm{~h}$ the cells reached a confluency of about $95 \%$.

\section{Instruments}

Two distinct biosensor systems, Epic $($ $)$ and a high resolution version of Epic $(\mathbb{R})$ BT (Corning Incorporated), were used. Epic $(\mathbb{R})$ system is a wavelength interrogation reader system. ${ }^{30}$ This system consists of a temperature-control unit, an optical detection unit, and an on-board liquid handling unit operated by robotics. The detection unit is centred on integrated fibre optics which scans across the plate with a temporal resolution of 15 s. The high resolution Epic $\mathbb{R}$ BT system is a swept wavelength interrogation system which uses a light beam from a swept tunable light source to illuminate the $3 \times 4$ biosensor array within a 384 well microplate biosensor bottom insert, and a high speed complementary metal-oxide semiconductor (CMOS) digital camera to record the escaped and reflected resonant lights with a spatial resolution of $12 \mu \mathrm{m} .{ }^{28,29,31}$ The tunable light source sweeps the wavelength range from 825 to $840 \mathrm{~nm}$ in a stepwise fashion. A total of 150 spectral images were acquired within a single sweeping cycle (3s), and were then processed into sensor resonance wavelength or DMR image in real time with a temporal resolution of $3 \mathrm{~s}$. The Epic $(\mathbb{R})$ system is tailored for resonant waveguide grating (RWG) biosensors in microplate, and is a bulky standalone system compatible to high throughput screening. The Epic $\mathbb{R}$ BT system is flexible in terms of biosensor footprint and 
operational procedure, and has a small footprint which can be placed inside cell culture incubators.

The RWG biosensor employs a surface bound evanescent wave, an electromagnetic field created by the total internal reflection of resonant light at a solution-surface interface which typically extends a short distance $(\sim 150 \mathrm{~nm})$ into the solution or adherent cells, to monitor in real-time ligandinduced dynamic redistribution of cellular matters within the bottom portion of cells. ${ }^{24,25}$ The DMR signal is often recorded as a time-series of the shifts (in picometers, pm) of the biosensor resonance wavelength. ${ }^{27}$ The resonance wavelength is a function of the local refractive index at or near the sensor surface, which is proportional to the mass density and distribution of biomaterials within the cells. ${ }^{24}$ Receptor signalling is known to result in protein trafficking, microfilament remodelling, cell adhesion alterations, and morphological changes of cells, all of which can lead to significant mass redistribution, thus contributing to the DMR signal obtained. Thus, a ligand-induced DMR signal is an integrated cellular response and provides a holistic view of receptor signalling and ligand pharmacology. ${ }^{27}$ The biosensor uses long-wavelength light for illumination of the biosensor but not the cells, and non-invasively monitors the DMR in real time.

\section{DMR assays}

Three different types of DMR assays, persistent assay in microplate, inhibitor washout assay in microplate, and perfusion assay under microfluidics, were used to elucidate the whole cell phenotypic efficacy of EGFR inhibitors. For DMR assay in microplate, an inhibitor was added to and incubated with the cells for $1 \mathrm{~h}$ and the cells were then challenged with EGF in the presence of the inhibitor. The preincubation step used was due to the slow cell uptake rate of TKIs.

For inhibitor washout DMR assay in microplate an inhibitor was added to and incubated with the cells for $1 \mathrm{~h}$, followed by washing out the free inhibitor in solution with the assay buffer five times using a plate washer (Bio-Tek Microplate Washers ELx $405^{\mathrm{TM}}$, Bio-Tek, Winooski, VT), and incubating with the assay buffer for $1 \mathrm{~h}$ and finally stimulating with EGF.

For perfusion DMR assay under microfluidics the cells were perfused sequentially with an inhibitor, then the assay buffer, and finally EGF. The inlet holes of the microfluidic biosensor device were connected to three independently operated syringes using Tygon Ò tubing, so that continuous perfusion of cells with three different solutions, one at a time, is possible without introducing any abrupt changes of shear stress or laminar flow perturbation inside the microfluidic chamber. The flow rate was $1 \mu \mathrm{l} \mathrm{min}{ }^{-1}$ for all solutions to minimize the effect of shear stress on cellular status. ${ }^{28,29}$

All real-time DMR signals were reported as a 2 min baseline right before EGF stimulation followed by a real time EGFinduced response. All DMR signals were obtained at $26{ }^{\circ} \mathrm{C}$ and were background corrected. It is worth noting that the EGFR activation in A431 under physiological condition gave rise to a DMR similar to that obtained at room temperature. ${ }^{31}$ For DMR assays in microplate, an averaged DMR response was obtained from the cells within the scanning path. ${ }^{32}$ For DMR assay under microfluidics an averaged response from the cells located within the central area $(0.2 \times 2 \mathrm{~mm})$ of a biosensor. $^{28,29}$ The assay buffer for all was $1 \times$ Hank's balanced salt solution, $20 \mathrm{mM}$ Hepes, pH 7.1 (HBSS). All studies were carried out with at least three replicates unless specifically mentioned.

\section{Data analysis}

All experimental data were analysed by using GraphPad Prism 5.0 (GraphPad Software Inc., San Diego, CA, USA). The $\mathrm{EC}_{50}$ and $\mathrm{IC}_{50}$ values were obtained by fitting the dose DMR response curves with nonlinear regression.

\section{Results and discussion}

We chose to study the whole cell efficacy of three EGFR inhibitors, gefitinib, erlotinib and AG1478. These TKIs are synthetic and quinazoline-derived small molecules that bind to the intracellular tyrosine kinase domain of EGFR, and inhibit EGF-induced receptor phosphorylation by competing for the intracellular MgATP binding site. We characterized the pharmacology of these TKIs in two native cell lines, A431 and HT-29. A431 expresses a high amount of EGFR and a low level of HER $2,{ }^{33}$ while HT-29 expresses moderate EGFR, a relatively high amount of HER2, and a low level of erbB4. ${ }^{34,35}$ We chose DMR assays under three distinct operational schemes ${ }^{19}$ to assess the molecular mechanism of action of these TKIs (Fig. 1). Persistent DMR assay in microplate probes the TKI allosteric modulation of EGF binding and the inhibition of receptor signaling at the whole cell level. Inhibitor washout DMR assay examines the effect of cell uptake and retention of these TKIs on receptor signaling. Perfusion DMR under microfluidics examines the functional recovery of receptor signaling after perfusion away both free and effluxed TKIs.

We first characterized the DMR of EGF in both A431 and HT-29 cells. Results showed that in A431 cells, EGF of $32 \mathrm{nM}$

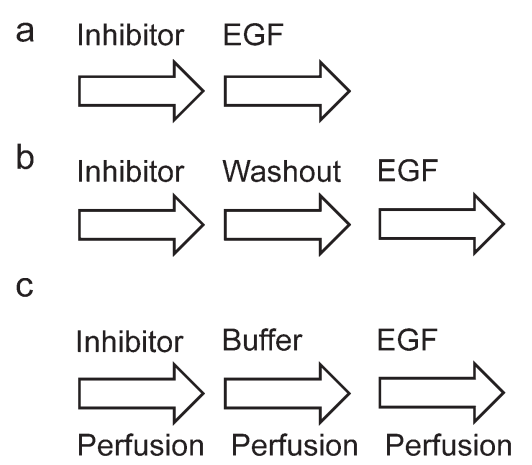

Fig. 1 Three distinct types of DMR assays. (a) Persistent DMR assay in microplate wherein the inhibitor is introduced before EGF stimulation, but remains throughout the assay. (b) Inhibitor washout DMR assay in microplate wherein after cell uptake the free inhibitor in solution is washed out. (c) Perfusion DMR assay under microfluidics wherein after cell uptake the free and effluxed inhibitor is perfused away. For all three assays the EGF response is obtained by monitoring cell responses in real time when EGF is always present once introduced. 
a
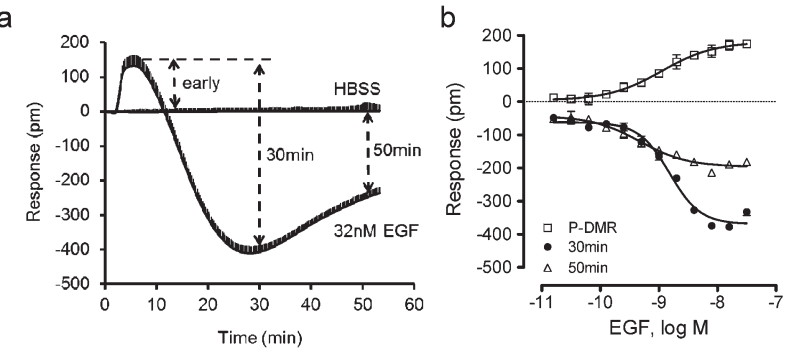

C

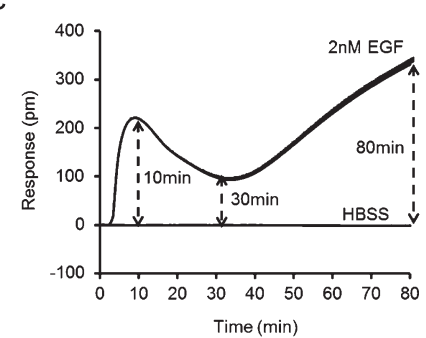

d

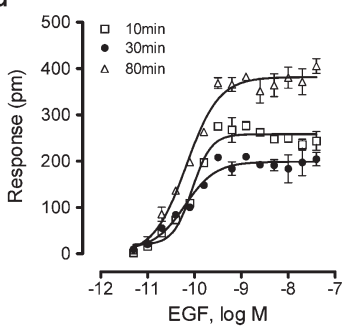

Fig. 2 The EGF DMR in A431 and HT-29. (a) The DMR of 32 nM EGF in A431 in comparison with the negative control (the assay buffer, HBSS). (b) The DMR amplitudes at 3, 30 and 50 min poststimulation as a function of EGF dose in A431. (c) The DMR of 2 nM EGF in HT-29 in comparison with the negative control (HBSS). (d) The DMR amplitudes at 10, 30 and 80 min poststimulation as a function of EGF dose in HT-29. Data represents mean \pm s.d. $(n=32,4,32$, and 4 for $a, b, c$, and d, respectively).

triggered a robust DMR consisting of three phases: an initial increased DMR event (positive-DMR, P-DMR), a decayed DMR event (negative-DMR, N-DMR) and another slowly increased DMR event (recovery positive-DMR, RP-DMR) (Fig. 2a). As the negative control, the cell response to the buffer only was also included. Fitting the dose response curve with nonlinear regression gave rise to an $\mathrm{EC}_{50}$ value that is a little sensitive to the EGF treatment time. The $\mathrm{EGF}^{\mathrm{EC}_{50}}$ was found to be $1.12 \pm$ $0.09,1.52 \pm 0.10$, and $0.48 \pm 0.04 \mathrm{nM}(n=4)$ for the DMR at 3 , 30 and $50 \mathrm{~min}$ post EGF stimulation, respectively (Fig. 2b). Similarly, in HT-29 cells, EGF of $2 \mathrm{nM}$ triggered a robust DMR which also consists of three phases: an initial P-DMR, an N-DMR and a RP-DMR (Fig. 2c). Compared to A431, the EGF DMR in HT-29 is distinct in that the decayed N-DMR in HT-29 does not reach a negative value relative to the baseline, and the kinetics for all three phases is slower, possibly due to the different expression pattern of the receptors. The dose response curves in HT-29 also yielded to a large degree an assay time-insensitive $\mathrm{EC}_{50}$, which was $89.4 \pm 7.1,62.3 \pm 5.8$, and $66.9 \pm 5.2 \mathrm{pM}(n=4)$ for the DMR at 10, 30 and $80 \mathrm{~min}$ post EGF stimulation, respectively. It is known that EGFR activation triggers multiple pathways; distinct pathways have different kinetics, and the EGF DMR represents an integration of these pathways into a real time kinetic response. Our previous pathway deconvolution studies have shown that for the EGF DMR in A431, the early P-DMR is mostly due to protein kinase C pathway, while the N-DMR is due to receptor internalization and mostly cell detachment, and the RP-DMR due to the re-attachment. ${ }^{36,37}$ This was confirmed by recent studies using quartz crystal microbalance with dissipation monitoring, a biosensor that is also sensitive to mass redistribution. ${ }^{38,39}$ However, the exact cellular mechanism(s) for the different DMR in HT-29 is mostly unknown. Considering that each phase of the EGF DMR contains information related to distinct pathways, we chose the abovementioned three time points to quantify the pharmacology of the TKIs.

Next, we compared the inhibition profiles of three TKIs in A431 under two different conditions: persistent treatment and inhibitor washout. For both, the inhibitor was used to pretreat the cells for $1 \mathrm{~h}$, given that cell uptake requires time to reach equilibrium. $^{40}$ The washout period was set to be $1 \mathrm{~h}$, considering that the cells were sensitive to buffer washing and took time $(\sim 30 \mathrm{~min})$ to reach steady state for biosensor measurement. ${ }^{19}$ Results showed that under the persistent treatment all inhibitors dose-dependently inhibit the EGF DMR (Fig. 3a,e,i). For the EGF responses at 30 and $50 \mathrm{~min}$, gefitinib gave rise to a clear biphasic modulation profile-it initially increased their amplitudes (that is, being more negative) followed by an inhibition (Fig. 3b); however, both erlotinib and AG-148 led to a monophasic inhibition (Fig. 3f and $\mathrm{j}$, respectively). This is possibly due to the fact that gefitinib can dose-dependently increase the binding affinity of EGF to the receptor in A431, and/or probably by increasing the numbers of EGFR dimers. ${ }^{33}$ Therefore, only until sufficiently high doses are reached inside the cells gefitinib starts to inhibit the EGF response.

Given that the inhibition curves of the EGF early DMR do not seem lead to good nonlinear regression fit, we used the late two events for $\mathrm{IC}_{50}$ determination. The apparent $\mathrm{IC}_{50}$ values to inhibit the EGF responses at 30 and $50 \mathrm{~min}$, respectively, was found to be $2.36 \pm 0.21$ and $2.86 \pm 0.18 \mu \mathrm{M}$ $(n=4)$ for gefitinib; $1.94 \pm 0.15$ and $2.79 \pm 0.24 \mu \mathrm{M}(n=4)$ for erlotinib; and $0.42 \pm 0.04$ and $0.48 \pm 0.03 \mu \mathrm{M}(n=4)$ for AG1478. These apparent $\mathrm{IC}_{50}$ values were generally much higher than their corresponding in vitro binding affinities, ${ }^{21}$ but consistent with many cell-based results. ${ }^{41}$

Unexpectedly, the inhibitor washout only had small impact on the dose-dependent inhibition of all three inhibitors (Fig. 3c,g,k). However, three noticeable differences were observed for these dose inhibition curves (Fig. 3d,h,l). First, all three inhibitors gave rise to a monophasic inhibition. Second, the washout unexpectedly increased the potency of gefitinib and AG-1478, but not erlotinib, to inhibit the response at $30 \mathrm{~min}$. The apparent $\mathrm{IC}_{50}$ values to inhibit the EGF responses at 30 and $50 \mathrm{~min}$, respectively, were found to be $0.80 \pm 0.07$ and $2.55 \pm 0.23 \mu \mathrm{M}(n=4)$ for gefitinib; $2.37 \pm$ 0.18 and $2.60 \pm 0.27 \mu \mathrm{M}(n=4)$ for erlotinib; and $0.13 \pm 0.01$ and $0.11 \pm 0.01 \mu \mathrm{M}(n=4)$ for AG-1478. Third, the Hill slope was less steep for all inhibitors under the washout condition than that under persistent treatment condition. This suggests that after the free inhibitors in solution are removed, the TKIs taken up by cells mostly remain inside cells and continuously suppress the EGFR signaling. Furthermore, the continuous presence of free TKIs may be important for the enhanced 
a

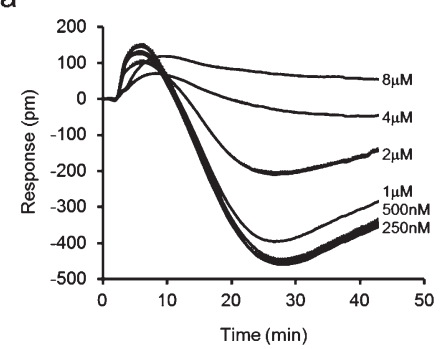

e

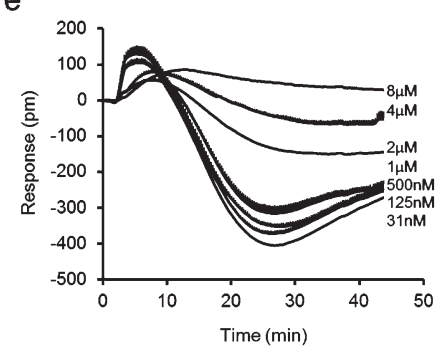

i

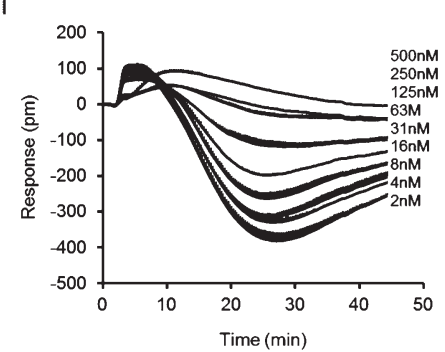

b

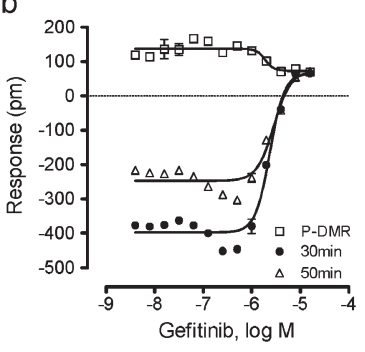

f

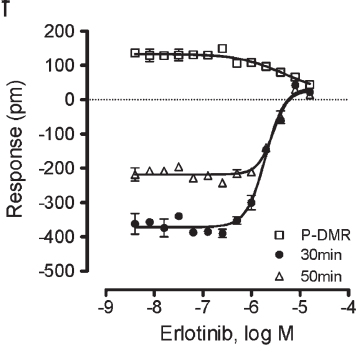

c

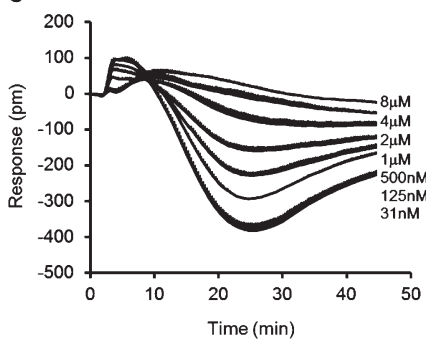

d

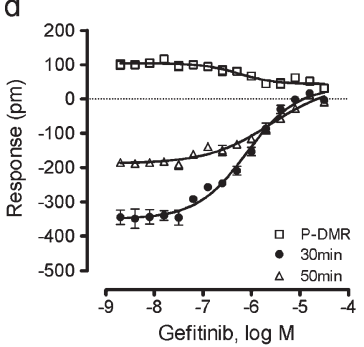

g

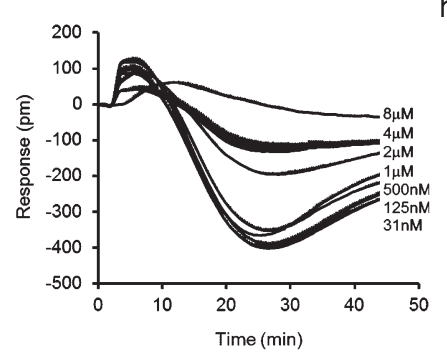

h

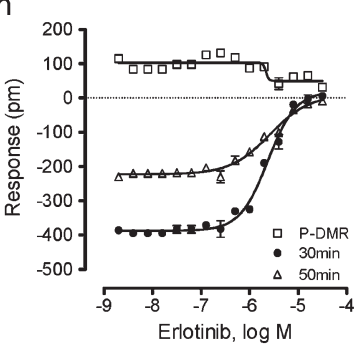

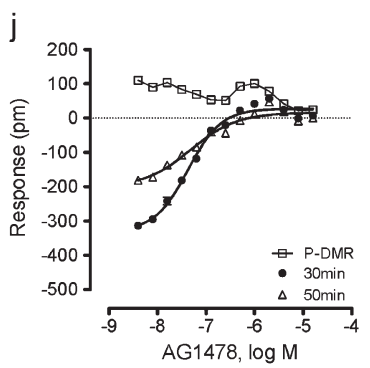

k

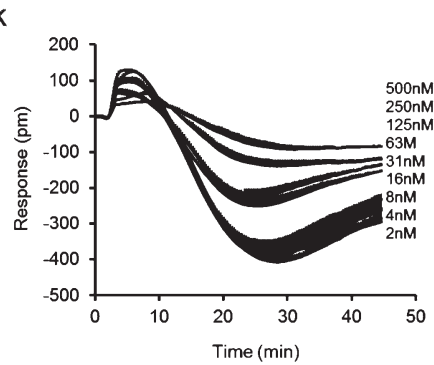

।

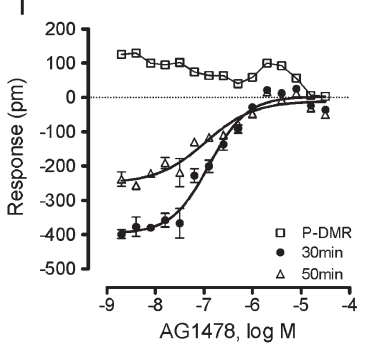

Fig. 3 Inhibition profiles of three TKIs in A431 under two different conditions. (a, e, i) The EGF DMR after 1 h pretreatment with gefitinib (a), erlotinib (e) and AG-1478 (i) at different doses. $(b, f, j)$ The EGF DMR amplitudes at 3, 30 and 50 min poststimulation as a function of the dose of gefitinib (b), erlotinib ( $f$ ) and AG-1478 (j) under persistent treatment condition. ( $c, g, k)$ The DMR of $32 \mathrm{nM}$ after pretreatment with gefitinib (c), erlotinib ( $\mathrm{g}$ ) and AG-1478 (k) at different doses for one hour followed by washout and one hour buffer incubation. ( $d, h, l)$ The EGF DMR amplitudes at 3, 30 and 50 min poststimulation as a function of the dose of gefitinib (d), erlotinib (h) and AG-1478 (I) under inhibitor washout condition. The EGF concentration was $32 \mathrm{nM}$ for all. Data represents mean \pm s.d. ( $n=4$ for all).

binding affinity of EGF to the receptor, thus leading to the need of high doses of TKIs to fully inhibit the receptor signaling when the EGF concentration is fixed.

Next, we compared the inhibition profiles of three TKIs in HT29 under persistent treatment and inhibitor washout conditions. Results showed that under both conditions all TKIs dose-dependently and mostly monotonically inhibited the DMR responses of $2 \mathrm{nM}$ EGF at 10 or $80 \mathrm{~min}$; however, they gave rise to a clear biphasic modulation profile of the EGF response at $30 \mathrm{~min}$ poststimulation, an initial potentiation followed by an inhibition, as the dose of all three TKIs increases (Fig. 4). Again, this is possibly due to the increased binding affinity of EGF to the receptors in the presence of TKIs. Unlike A431, the EGF DMR modulation patterns by all three TKIs were almost identical between the two different assay formats. For persistent treatment, the apparent $\mathrm{IC}_{50}$ values to inhibit the EGF responses at 10 and $80 \mathrm{~min}$, respectively, were found to be about 7.6 and $7.5 \mu \mathrm{M}(n=4$; only the mean value given due to the high Hill slope) for gefitinib; $10.6 \pm 0.9$ and $11.7 \pm 1.0 \mu \mathrm{M}(n=4)$ for erlotinib; and $0.48 \pm$
0.04 and $0.34 \pm 0.03 \mu \mathrm{M}(n=4)$ for AG-1478 (Fig. 4b,f,j). The steepness of the dose inhibition curves was the greatest for gefitinib with a Hill slope of 16 to 18 , moderate for erlotinib with a Hill slope of 3.2 to 3.9, and the smallest for AG-1478 with a Hill slope of 1.9 to 2.1. For TKI washout, the apparent $\mathrm{IC}_{50}$ values to inhibit the EGF responses at 10 and $80 \mathrm{~min}$, respectively, were found to be about $8.6 \pm 0.7$ and $6.8 \pm 0.5$ $\mu \mathrm{M}(n=4)$ for gefitinib; 16.0 and $15.8 \mu \mathrm{M}(n=4)$ for erlotinib; and $0.91 \pm 0.07$ and $0.51 \pm 0.04 \mu \mathrm{M}(n=4)$ for AG-1478 (Fig. 4d,h,l). The Hill slope was 3.0 and 4.5 for gefitinib, 12 and 26 for erlotinib, and 1.5 and 2.0 for AG-1478. Also noticeably, but different from A431, was that for the EGF response at 10 and $80 \mathrm{~min}$, all TKIs first slightly increased the signal followed by inhibition, as their doses increase. The apparent $\mathrm{IC}_{50}$ and Hill slope patterns of TKIs were distinct from A431, possibly due to the different expression patterns of different EGFR family members in the two cells. Nonetheless, the ability of TKI pretreatment to block EGFR signaling after inhibitor washout suggests that TKIs were taken up by cells and mostly remain inside cells. 
a

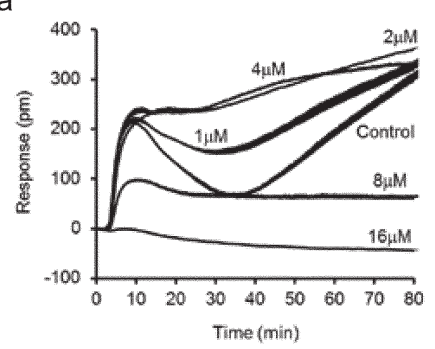

e

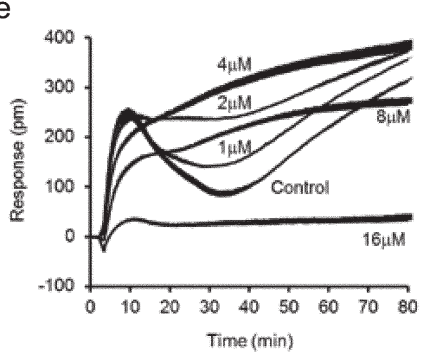

i

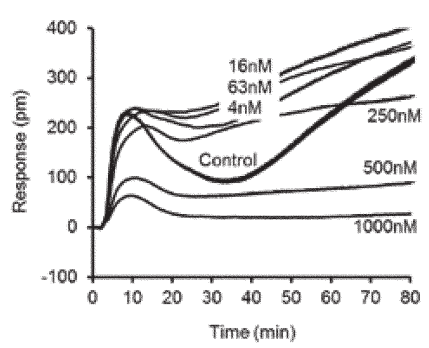

b

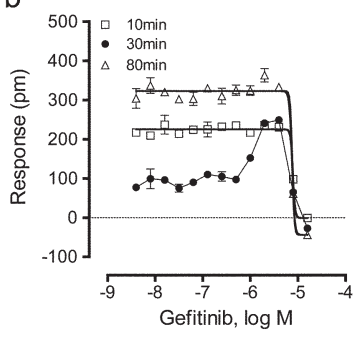

$f$

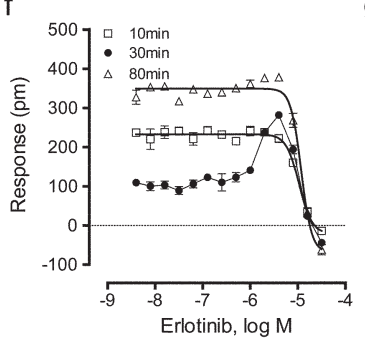

j

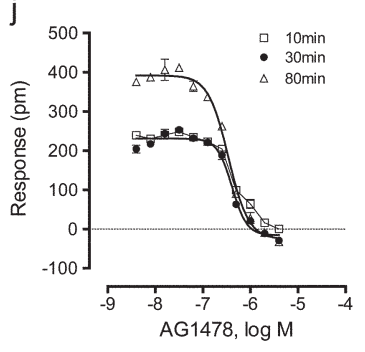

c

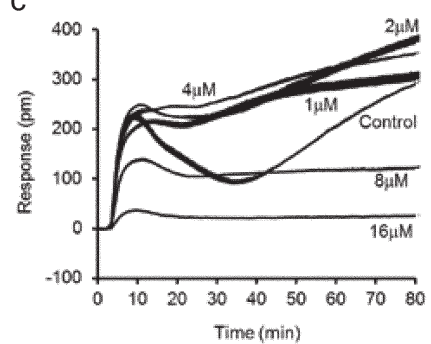

g

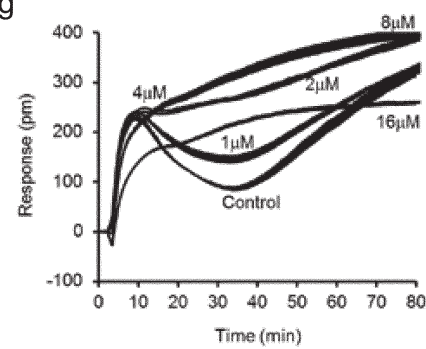

k

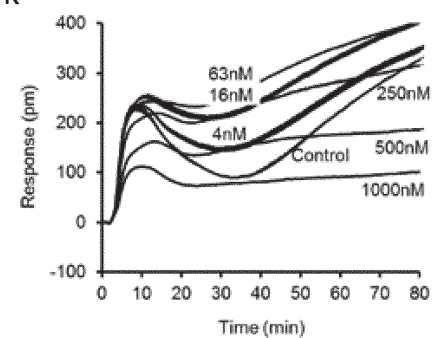

d

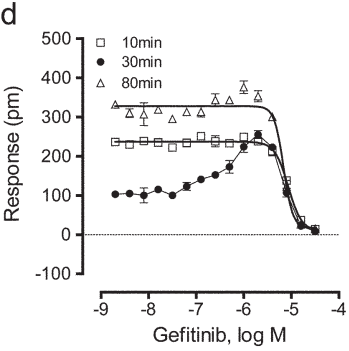

h

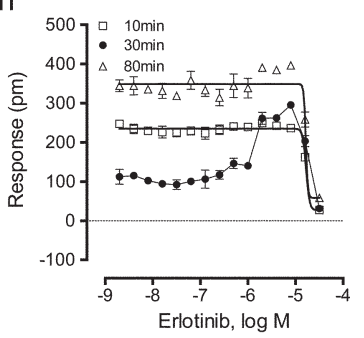

I

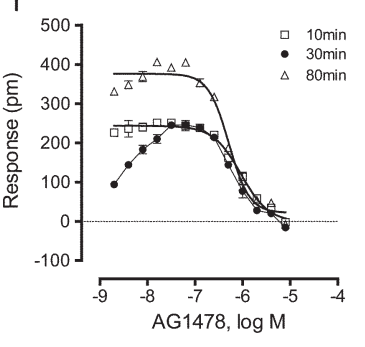

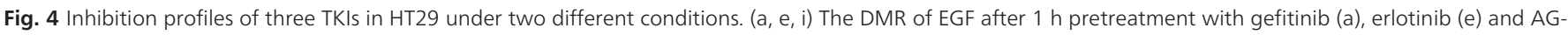

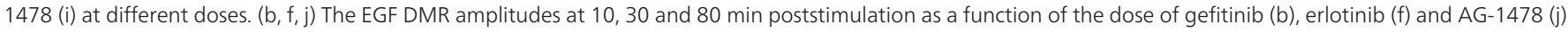

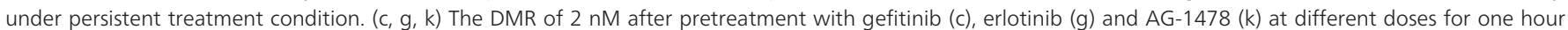

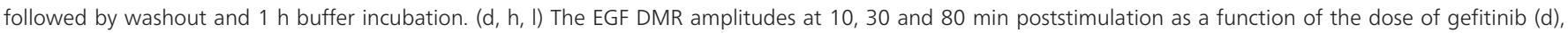
erlotinib (h) and AG-1478 (I) under inhibitor washout condition. The EGF concentration was 2 nM for all. Data represents mean \pm s.d. $(n=4$ for all).

Lastly, we examined the effect of TKI removal by perfusion using DMR assay under microfluidics. This assay permits complete removal of both free and effluxed inhibitors. The EGF response in the presence of an equal amount of an inhibitor after the initial perfusion with the inhibitor was used as a positive control; this condition is similar to the persistent DMR in microplate, except that there is a slow and continuous perfusion throughout the assay. Results showed that in A431, an EGF of $32 \mathrm{nM}$ triggered a DMR similar to that obtained in microplate, suggesting that the continuous perfusion at the slow rate used had only a small effect on the EGFR signaling in this cell line (Fig. 5a). The persistent perfusion with $10 \mu \mathrm{M}$ gefitinib almost completely inhibited the EGF response, while the cells responded to EGF with a small but noticeable DMR when a perfusion step with the buffer was introduced between the first TKI and the last EGF stimulation steps (Fig. 5a). The EGF DMR was greater when the buffer perfusion step lasted longer, suggesting that some intracellular inhibitors are released and removed during the buffer perfusion step. Almost identical patterns were observed for erlotinib (Fig. 5b). In contrast, the persistent perfusion with sotalol completely inhibited the isoprotenerol DMR; but the buffer perfusion of $10 \mathrm{~min}$ was found to be sufficient to abolish the antagonistic effect of sotalol on the isoprotenerol-induced DMR (Fig. 5c). The isoprotenerol DMR in A431 has been shown to be due to the activation of endogenous $\beta_{2}$-adrenergic receptor, a prototypic G protein-coupled receptor (GPCR). ${ }^{42,43}$ Sotalol is a known $\beta$-blocker. ${ }^{44}$ This suggests that sotalol is a reversible competitive antagonist with a rapid off rate for the receptor. Unlike EGFR TKIs which bind to the intracellular domain of the receptor after getting inside the cell first, most GPCR antagonists bind to the site(s) located within the transmembrane domains of the receptor directly from the extracellular side.

In HT-29 cells, perfusion with 2 nM EGF triggered a biphasic DMR that is somewhat distinct from that obtained in microplate (comparing Fig. 5d with Fig. 2c), suggesting that the continuous perfusion had a more pronounced impact on the EGFR signaling in HT-29 cells than that in A431 cells. The continuous perfusion with $10 \mu \mathrm{M}$ gefitinib significantly suppressed the EGF DMR, and the buffer perfusion clearly, but not completely, reversed the inhibitory effect of gefitinib 
a

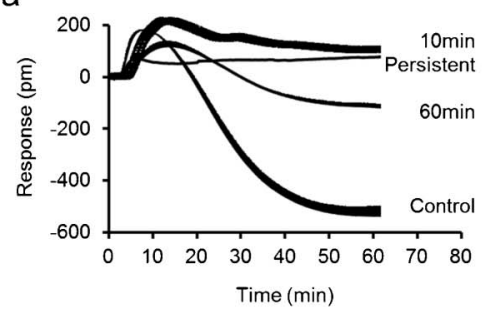

d

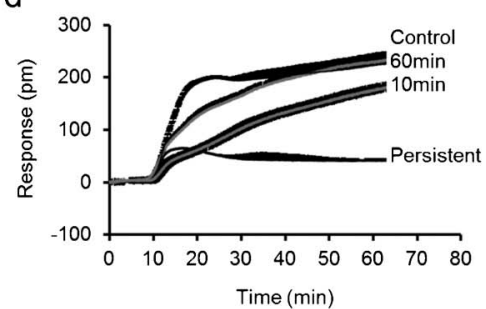

b

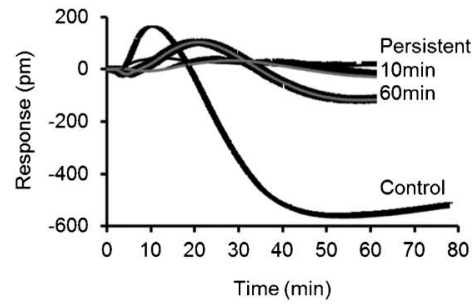

e

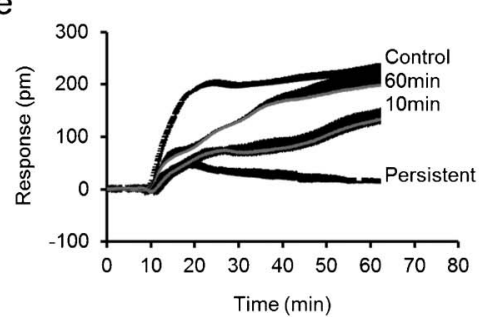

c

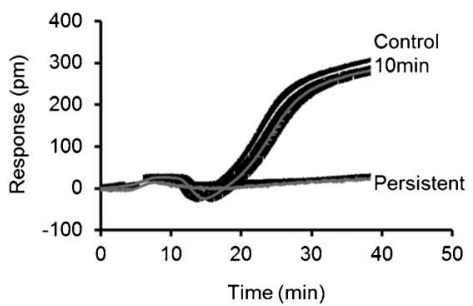

f

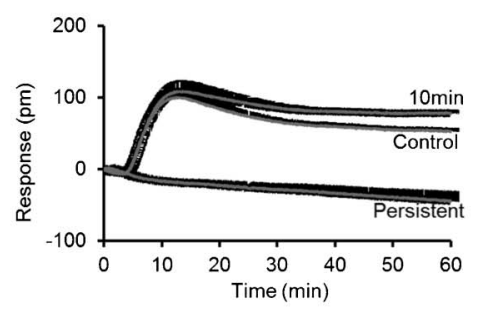

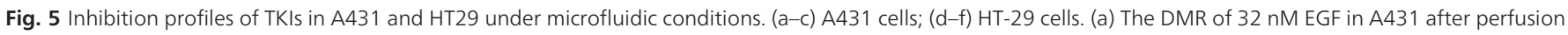

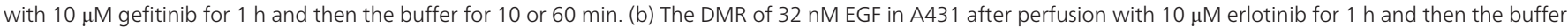

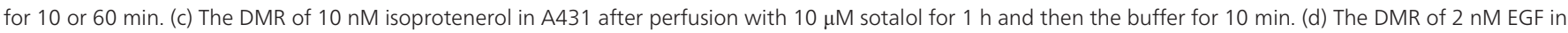

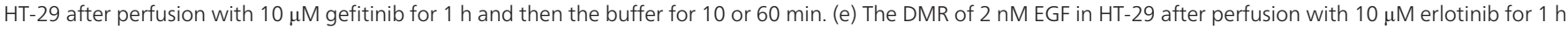

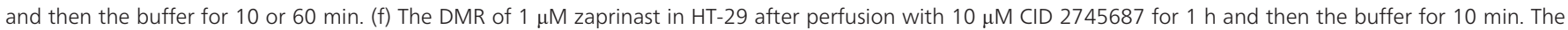

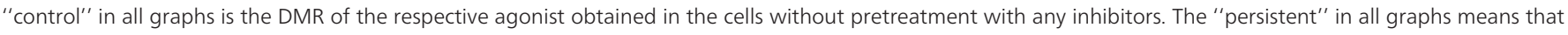
the DMR of the respective agonist was obtained after perfusion with the corresponding antagonist for $1 \mathrm{~h}$ followed by the mixture of the agonist and equal concentration of the antagonist. Data represents mean \pm s.d. ( $n=3$ for all).

on the EGF DMR (Fig. 5d). A similar pattern was observed for erlotinib (Fig. 5e). As a control, the persistent perfusion with $10 \mu \mathrm{M}$ CID 2745687 completely suppressed the DMR signal of $1 \mu \mathrm{M}$ zaprinast in HT-29, when the buffer perfusion of $10 \mathrm{~min}$ was sufficient to eliminate the inhibitory effect of CID 2745687 (Fig. 5f). CID 2745687 is a known GPR35 antagonist, ${ }^{45,46}$ and zaprinast is a known GPR35 agonist. ${ }^{47}$ The zaprinast DMR in HT-29 was previously shown to be due to the activation of endogenous GPR35. ${ }^{48,49}$ This result suggests that CID 2745687 is a reversible antagonist with a fast off rate for the GPR35.

The whole cell phenotypic pharmacology of EGFR TKIs is complicated by several factors (Fig. 6). First, EGFR behaves as an allosteric protein in that the agonists bind to the extracellular domain of the receptor and result in the receptor dimerization, while the TKIs bind to the intracellular protein tyrosine kinase domain of the receptor. The binding of TKIs has been shown to alter the binding characteristics of EGF to the EGFR in A431. ${ }^{33}$ Therefore, the inhibition profile of the whole cell EGF response by these TKIs reflect an allosteric, rather than directly competitive, effect between the two types of ligands. This may at least partially explain the differences in the Hill slopes of the inhibition profiles observed, as well as the biphasic inhibition profiles of some TKIs under some conditions. Second, the expression pattern of EGFR family members in the two cell lines is different. ${ }^{33-35}$ The EGFinduced heterodimerization may contribute to the difference in both the EGF DMR and the TKI inhibition profiles. Third, unlike GPCR ligands which bind to the receptor from the extracellular side, the TKIs need be taken up by the cells first. Cell uptake of TKIs is a relatively slow process via both active and passive processes, taking over $30 \mathrm{~min}$ to reach a plateau in A431 and other cells. ${ }^{40,50}$ The intracellular TKI concentration is dependent on the amount of TKIs added and the time of cell uptake, but is generally higher than that in the assay solution. This obviously impacts the characteristics of the TKI inhibition curves. Fourth, the retention of TKIs inside the cells once

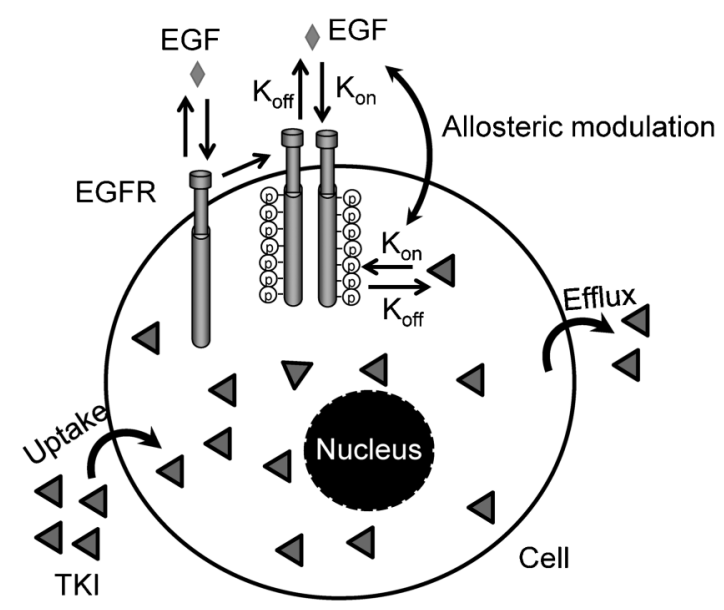

Fig. 6 The factors influencing the whole cell phenotypic efficacy of TKIs. The intracellular pool of TKIs is a function of both cell uptake and effluxing. Free TKIs in solution can be taken up by the cells via both passive (minor) and active (major) processes. The intracellular TKIs can be pumped out through transporters. The binding of TKIs to the tyrosine kinase domain can alter the binding affinity and kinetics of the EGF to the receptor. The TKI binding kinetics, in particular the off rate can also influence the efficacy. 
taken up also contributes to the overall efficacy of TKIs to block EGFR signaling. Kinase inhibitors are known to generate an "echo" effect when the cells are first exposed to the inhibitor and then washed to remove the free inhibitor in solution, ${ }^{51}$ suggesting the importance of cell retention on the efficacy of TKIs in cells. The cell retention of TKIs is a function of cell uptake and effluxing. The TKI effluxing can be accomplished through efflux transporter such as ABCG2. All three TKIs tested are ABCG2 substrates; the overexpression of ABCG2 has been shown to protect A431 cells from death after gefitinib through a mechanism involving pumping the TKIs out. ${ }^{52,53}$ Furthermore, gefitinib at high doses is also an ABCG2 inhibitor. The native A431 cells express little ABCG2; ${ }^{52}$ however, the native HT-29 expresses high amount of ABCG2.$^{54}$ This is consistent with our data showing that the buffer perfusion led to faster recovery of the EGF DMR in HT29 than that in A431 cells; the buffer perfusion is more effective to cause the recovery of the EGF signal in HT-29 cells than the inhibitor washout in plates; and the potency of three TKIs is generally higher in A431 than HT-29 cells. Lastly, we cannot rule out the impact of the binding kinetics on the whole cell inhibition profiles of TKIs. All three TKIs tested are reversible inhibitors for EGFR but with distinct affinity and kinetics. ${ }^{21}$ The TKI with a slower off rate may display a higher efficacy to block the EGFR signaling. However, considering the rapid off rate of the three TKIs tested ${ }^{21}$ as well as the slow cell uptake ${ }^{40,50}$, both on and off rates are obviously less important than the cell uptake and retention. ${ }^{55}$

\section{Conclusions}

We have examined the label-free whole cell phenotypic efficacy of three EGFR inhibitors to block the receptor signaling in two native cell lines. DMR assays under three distinct operational schemes revealed that the drug uptake and retention seem have a dominating role in determining their whole cell efficacy. DMR assays, in particular those under microfluidics, may offer a unique means to determine the influence of efflux transporter on the efficacy of EGFR and other kinase inhibitors, and to probe the transporter-related drug resistance.

\section{Notes and references}

1 M. D. Marmor, K. B. Skaria and Y. Yarden, Int. J. Radiat. Oncol., Biol., Phys., 2004, 58, 903-913.

2 K. Oda, Y. Matsuoka, A. Funahashi and H. Kitano, Mol. Syst. Biol., 2005, 1, 2005.0010.

3 H. Zhang, A. Berezov, Q. Wang, G. Zhang, J. Drebin, R. Murali and M. I. Greene, J. Clin. Invest., 2007, 117, 2051.

4 C. L. Arteaga, Oncologist, 2002, 4, 31.

5 M. Ono and M. Kuwano, Clin. Cancer Res., 2006, 12, 7242. 6 J. G. Paez, P. A. Jänne, J. C. Lee, S. Tracy, H. Greulich, S. Gabriel, P. Herman, F. J. Kaye, N. Lindeman, T. J. Boggon, K. Naoki, H. Sasaki, Y. Fujii, M. J. Eck, W.
R. Sellers, B. E. Johnson and M. Meyerson, Science, 2004, 304, 1497.

7 R. Sordella, D. W. Bell, D. A. Haber and J. Settleman, Science, 2004, 305, 1163.

8 F. Ciardiello and G. N. Tortora, N. Engl. J. Med., 2008, 358, 1160.

9 F. Tan, X. Shen, D. Wang, G. Xie, X. Zhang, L. Ding, Y. Hu, W. He, Y. Wang and Y. Wang, Lung Cancer, 2012, 76, 177.

10 N. E. Hynes and H. A. Lane, Nat. Rev. Cancer, 2005, 5, 341.

11 D. M. Jackman, V. A. Miller, L. A. Cioffredi, B. Y. Yeap, P. A. Jänne, G. J. Riely, M. G. Ruiz, G. Giaccone, L. V. Sequist and B. E. Johnson, Clin. Cancer Res., 2009, 15, 5267.

12 S. Kobayashi, T. J. Boggon, T. Dayaram, R. A. Jänne, O. Kocher, M. Meyerson, B. E. Johnson, M. J. Eck, D. G. Tenen and B. Halmos, N. Engl. J. Med., 2005, 352, 786.

13 M. Fakih and M. Vincent, Curr. Oncol., 2010, 17(Suppl. 1), S18.

14 C. Lemos, G. Jansen and G. J. Peters, Br. J. Pharmacol., 2008, 98, 857.

15 P. Borst, Open Biology, 2012, 2, 120066.

16 D. C. Swinney, Nat. Rev. Drug Discovery, 2004, 3, 801.

17 R. A. Copeland, D. L. Pompliano and T. D. Meek, Nat. Rev. Drug Discovery, 2006, 5, 730.

18 D. C. Swinney, Annu. Rep. Med. Chem., 2011, 46, 301.

19 H. Deng, C. Wang, M. Su and Y. Fang, Anal. Chem., 2012, 84, 8232 .

20 D. Guo, T. Mulder-Krieger, A. P. Ijzerman and L. H. Heitman, 2012, 166, 1876.

21 E. R. Wood, A. T. Truesdale, O. B. McDonald, D. Yuan, A. Hassel, S. H. Dickerson, B. Ellis, C. Pennisi, E. Horne, K. Lackey, K. J. Alligood, D. W. Rusnak, T. M. Gilmer and L. Shewchuk, Cancer Res., 2004, 64, 6652.

22 M. Goyal, M. Rizzo, F. Schumacher and C. F. Wong, J. Med. Chem., 2009, 52, 5582.

23 S. Bairy and C. F. Wong, Proteins: Struct., Funct., Bioinf., 2011, 79, 2491.

24 Y. Fang, A. M. Ferrie, N. H. Fontaine, J. Mauro and J. Balakrishnan, Biophys. J., 2006, 91, 1925.

25 Y. Fang, Drug Discovery Today: Technol., 2010, 7, e5-e11.

26 T. Kenakin, Nat. Biotechnol., 2010, 28, 928.

27 Y. Fang, Expert Opin. Drug Discovery, 2011, 6, 1285.

28 V. Goral, Q. Wu, H. Sun and Y. Fang, FEBS Lett., 2011, 585, 1054.

29 V. Goral, Y. Jin, H. Sun, A. M. Ferrie, Q. Wu and Y. Fang, PLoS One, 2011, 6, e19282.

30 G. Li, A. M. Ferrie and Y. Fang, J. Assoc. Lab. Autom., 2006, 11, 181-187.

31 A. M. Ferrie, O. D. Deichmann, Q. Wu and Y. Fang, Appl. Phys. Lett., 2012, 100, 223701.

32 Y. Fang, Assay Drug Dev. Technol., 2006, 4, 583.

33 H. Bjorkelund, L. Gedda, P. Barta, M. Malmqvist and K. Andersson, PLoS One, 6, e24739.

34 M. C. Prewett, A. T. Hooper, R. Bassi, L. M. Ellis, H. W. Waksal and D. J. Hicklin, Clin. Cancer Res., 2002, 8, 994.

35 H. Xu, Y. Yu, D. Marciniak, A. K. Rishi, F. H. Sarkar, O. Kucuk and A. P. Majumdar, Mol. Cancer Ther., 2005, 4, 435.

36 Y. Fang, A. M. Ferrie, N. H. Fontaine and P. K. Yuen, Anal. Chem., 2005, 77, 5720.

37 Y. Fang, Expert Opin. Drug Discovery, 2010, 5, 1237. 
38 J. Y. Chen, M. Li, L. S. Penn and J. Xi, Anal. Chem., 2011, 83, 3141.

39 J. Y. Chen, A. Shahid, M. P. Garcia, L. S. Penn and J. Xi, Biosens. Bioelectron., 2012, 38, 375.

40 M. R. Zhang, K. Kumata, A. Hatori, N. Takai, J. Toyohara, T. Yamasaki, K. Yanamoto, J. Yui, K. Kawamura, S. Koike, K. Ando and K. Suzuki, Mol. Imaging Biol., 2010, 12, 181.

41 G. K. Smith and E. R. Wood, Drug Discovery Today: Technol., 2010, 7, e13.

42 Y. Fang and A. M. Ferrie, FEBS Lett., 2008, 582, 558.

43 A. M. Ferrie, H. Sun and Y. Fang, Sci. Rep., 2011, 1, e33.

44 J. G. Baker, Br. J. Pharmacol., 2005, 144, 317.

45 P. Zhao, H. Sharir, A. Kapur, A. Cowan, E. B. Geller, M. W. Adler, H. H. Seltzman, P. H. Reggio, S. Heynen-Genel, M. Sauer, T. D. Y. Chung, Y. Bai, W. Chen, M. G. Caron, L. S. Barak and M. E. Abood, Mol. Pharmacol., 2010, 78, 560.

46 H. Hu, H. Deng and Y. Fang, PLoS One, 2012, 7, e34934.

47 Y. Taniguchi, H. Tonai-Kachi and K. Shinjo, FEBS Lett., 2006, 580, 5003.

48 H. Deng, H. Hu and Y. Fang, FEBS Lett., 2011, 585, 1957.
49 H. Deng, H. Hu, M. He, J. Hu, W. Niu, A. M. Ferrie and Y. Fang, J. Med. Chem., 2011, 54, 7385.

50 M. Galetti, R. R. Alfieri, A. Cavazzoni, S. L. Monica, M. Bonelli, C. Fumarola, P. Mozzoni, G. de Palma, R. Andreoli, A. Mutti, M. Mor, M. Tiseo, A. Ardizzoni and P. G. Petronini, Biochem. Pharmacol., 2010, 80, 179.

51 T. Ona and J. Shibata, Anal. Bioanal. Chem., 2010, 398, 2505.

52 N. B. Elkind, Z. Szentpetery, A. Apati, C. Ozvegy-Laczka, G. Varady, O. Ujhelly, K. Szabó, L. Homolya, A. Váradi, L. Buday, G. Kéri, K. Német and B. Sarkadi, Cancer Res., 2005, 65, 1770.

53 Z. Shi, S. Parmar, X.-X. Peng, T. Shen, R. W. Robey, S. E. Bates, L.-W. Fu, Y. Shao, Y.-M. Chen, F. Zang and Z.S. Chen, Oncol. Rep., 2009, 21, 483.

54 Y. Imai, M. Nakane, K. Kage, S. Tsukahara, E. Ishikawa, T. Tsuruo, Y. Miki and Y. Sugimoto, Mol. Cancer Ther., 2002, 1, 611.

55 C. A. Faaland, F. H. Mermelstein, J. Hayashi and J. D. Laski, Mol. Cell. Biol., 1991, 11, 2697. 